\title{
Developing the Multidimensional Visual Scale Assessing Affect, Anxiety, Pride and Energy (AAPE) through a Research Partnership with Autistic Scholars
}

\author{
Ariana Riccio $^{1 *}$, Jin Delos Santos ${ }^{2}$, Steven K. Kapp ${ }^{3+}$, Allison Jordan ${ }^{4}$, Danielle DeNigris ${ }^{5}$,
} Kristen Gillespie-Lynch ${ }^{6}$

${ }^{1}$ Department of Psychology, The Graduate Center, CUNY, New York, NY, USA, ariccio@gradcenter.cuny.edu

${ }^{2}$ Department of Psychology, Hunter College, CUNY, New York, NY, USA, jindelsan@gmail.com

${ }^{3}$ Egenis, University of Exeter, Exeter, UK, steven.kapp@gmail.com

${ }^{4}$ Borough of Manhattan Community College and The College of Staten Island, CUNY, New York, NY, USA, allison.jordan8@gmail.com

${ }^{5}$ Department of Psychology, Fairleigh Dickinson University, Madison, NY, USA, ddenigris@gmail.com

${ }^{6}$ Department of Psychology, The Graduate Center and The College of Staten Island, CUNY, New York, NY, USA, kgillyn@gmail.com

\begin{abstract}
*Ariana Riccio is the corresponding author for this manuscript. She can be reached at ariccio@gradcenter.cuny.edu or $3655^{\text {th }}$ Ave., New York, NY 10016.

${ }^{+}$Dr. Kapp is now at the Department of Psychology, University of Portsmouth, Portsmouth, UK, steven.kapp@port.ac.uk
\end{abstract}

Keywords: emotion, measurement, visual scale, participatory research, autistic identity

\author{
Abstract word count: 248 \\ Manuscript word count: 5193 \\ Lay summary word count: 592
}


Authorship Confirmation Statement:

AR helped design this study, processed and analyzed the data, conducted the literature review, and wrote many drafts of this manuscript. This work will serve as one of four studies included in her doctoral dissertation. JDS was the undergraduate artist responsible for creating and editing all versions of each emotion dimension and served as the secondary qualitative data coder for all qualitative participant data. SKK (in collaboration with KGL) helped conceptualize the study design in discussions about the need for picture-based research measures while a doctoral student himself and has contributed to the literature review, scale development, and writing as a postdoctoral Research Fellow at the University of Exeter, supported by the Wellcome Trust, grant number 108676/Z/15/Z. AJ was involved in the development and iteration of scale dimensions and assisted in editing this manuscript. DD was involved in the design and iteration of scale dimensions and assisted in the editing of this manuscript. KGL, who is AR's doctoral advisor, developed the idea for this study with AR and SKK, played a leading role in designing the study, and contributed very substantially to the literature review and writing of the manuscript. All authors contributed significantly to participatory research group meetings in person or online and contributed their ideas and time to the development of this paper. All coauthors have reviewed and approve of the submission of this manuscript. This manuscript has been submitted solely to Autism in Adulthood and is not published, in press, or submitted elsewhere. 
Author Disclosure Statement

No competing financial interests exist. 


\begin{abstract}
Autism research studies have traditionally failed to represent the full diversity of the autism spectrum due to the lack of measures available for use with participants who prefer to express themselves visually. A multidimensional measure of emotions which can include both picture and text-based prompts may improve accessibility of emotion rating measures and broaden participation in research and educational evaluations to include those who communicate in diverse ways. Picture-based measures designed to assess participants' emotions may be useful for research concerning autistic identity and service evaluation, two areas where representation of diverse perspectives is needed. Our participatory group of autistic and non-autistic researchers developed a Multidimensional Scale Assessing Affect, Anxiety, Pride and Energy (AAPE) by adapting and expanding upon an existing emotion rating scale.

When testing the AAPE with autistic college students $(n=72)$, college students' openended responses indicated that the AAPE's dimensions of affect (97.2\% correct), anxiety (79.2\% correct), and energy ( $84.7 \%$ correct) were well comprehended without text-based labels with potential for improvement in how pride $(52.8 \%$ correct) was represented. When provided with the labels that each dimension was intended to represent, participants generally agreed that each emotional dimension was well-represented. When tested in an informal educational summer camp with autistic children and adolescents $(n=50)$, the AAPE was well-received and revealed insights about the students' emotional responses to different instructional strategies that can guide curricular improvements. The AAPE has utility as a tool to help diverse autistic individuals self-advocate and improve research and services.
\end{abstract}


Developing the Multidimensional Visual Scale Assessing Affect, Anxiety, Pride and Energy

(AAPE) through a Research Partnership with Autistic Scholars

\section{Introduction}

Studies assessing attitudes toward autism typically require participants to communicate through spoken or written language, greatly limiting representation of the full diversity of the autism constellation ${ }^{1}{ }^{2-5}$ Literature investigating topics such as autistic experiences, services, and quality of life often rely on caregivers to speak for those individuals deemed "lacking capacity to self-report." 6,7 Despite calls for increasing representation of people who have traditionally been underrepresented within autism research (e.g., people with co-occurring intellectual disabilities and people who do not speak), ${ }^{8,9}$ most existing research assessing the perspectives of autistic individuals has relied on purely language-based assessments..$^{10,11}$

Assessments that are accessible to individuals across the autism constellation are needed so that a more representative group of autistics may share their experiences using communication methods designed around their abilities and challenges. Given that autism is often associated with visual strengths, ${ }^{12}$ picture-based options may increase engagement with and/or comprehension of survey items. Indeed, assessments that include picture-based options have been found to be more engaging and accessible than purely text-based measures for young children and individuals with intellectual disabilities. ${ }^{13-16}$ Therefore, picture-based measures may also allow diverse autistic individuals, including people with co-occurring intellectual disabilities and people who do not speak or read, to participate in research and educational evaluations. Picture-based strategies such as photovoice and Talking Mats are increasingly (albeit still rarely) used to assess the first-person perspectives of autistic people with diverse communicative capacities. ${ }^{10,11}$ 
The current study presents the participatory process of developing an emotion rating scale, the Multidimensional Visual Scale Assessing Affect, Anxiety, Pride and Energy (AAPE), that is designed to be accessible to people who communicate and comprehend in diverse ways. This is the first stage of a larger ongoing project conducted by our participatory research group, which focuses on measuring aspects of autistic identity using a picture-based scale, with accompanying text-based item descriptions, to allow multiple routes of access.

We focused on developing an emotion rating scale because emotions are central to the development of identity and relationships yet it can be difficult for autistic people to recognize and express emotions. ${ }^{17-21}$ Although researchers have long speculated that autistic people have specific difficulties forming affective relationships and understanding emotions, ${ }^{22,23}$ more recent research and accounts from autistic people suggest that emotion recognition difficulties observed among some autistic people are attributable to language difficulties and/or co-occurring alexithymia (difficulty identifying and describing one's own emotions) rather than autism. ${ }^{24-27}$

An emotion rating scale that includes both pictures and words may provide more opportunities for autistic people with language difficulties and/or alexithymia to express their emotions than purely text-based rating scales. Indeed, during the review process for this paper, an autistic co-author (the artist who drew the AAPE) reported that he experiences alexithymia. Despite having highly advanced verbal skills, he finds it easier to identify emotions in pictures than words. Expressing himself through drawing has helped him learn to understand emotions more deeply. By developing a multimodal emotion rating scale, we hoped to provide autistic people with diverse communicative, emotion recognition, and cognitive skills with a tool they can use to express how they feel about their experiences so they can self-advocate and help improve available supports. 


\section{Learning from an Established Picture-Based Measure of Emotion}

The AAPE is inspired by one of the most widely used picture-based rating scales of emotions, the Self-Assessment Manikin (SAM; Figure 1). ${ }^{28}$ A fixture in emotion-related literature cited over 6,000 times, the SAM has been used by researchers to address numerous research questions, most commonly the neural correlates of emotions. Described as a "nonverbal pictorial assessment technique," the SAM provides participants with a 5-point scale for rating their emotional valence, arousal, and dominance using a gender- and context-neutral avatar. Contrasting with the viewpoint that people experience discrete and universal emotions (e.g., fear), the SAM frames emotions as responses to stimuli that vary along key dimensions (e.g., valence, arousal and control). ${ }^{29}$ The SAM has proven useful as a method of rating participants' feelings about various contexts and prompts. ${ }^{30,31}$ Our team became aware of the SAM after viewing a poster by a colleague who was adapting the SAM in collaboration with Deaf individuals during counseling sessions. ${ }^{32}$

\section{[Insert Figure 1]}

Although the SAM effectively captures multidimensional aspects of emotions, the illustrations used for the SAM may not be entirely intuitive. The widely cited paper establishing the validity of the SAM as a "non-verbal" assessment did not provide much detail about how the SAM was administered, instead noting that the SAM ratings used in the paper had been obtained using the International Affective Picture System (IAPS). ${ }^{28}$ The IAPS is a popular research tool containing emotionally evocative images that was developed by the creators of the SAM. The IAPS Technical Administration Manual instructs researchers to describe each of the SAM scales using a plethora of emotional vocabulary so that participants are able to use the SAM as intended by its creators. ${ }^{33}$ For example, the manual describes the excited terminus of the SAM's arousal 
scale using the words "stimulated, excited, frenzied, jittery, wide-awake, aroused" (p. 4) and the calm terminus using the words "relaxed, calm, sluggish, dull, sleepy, unaroused" (p. 4). The instructions that are read to participants before they use the SAM to rate the IAPS are one and a half pages long. In a recent study where the instructions for the SAM were adapted for use with 10 autistic adults, the instructions to help participants learn how to use the SAM were approximately 900 words long. ${ }^{31}$ While the designation of the SAM as a "non-verbal" assessment suggests that it may not require language to rate one's emotions, the lengthy verbal instructions which accompany the SAM suggest that the SAM requires substantial languagebased clarification before it can meaningfully be used.

\section{Existing Rating Scales Developed Using Participatory Processes}

To the best of our knowledge, only two other research teams have documented a participatory process wherein people with and without disabilities collaboratively adapted established measures to increase their accessibility for people with developmental disabilities. ${ }^{34,35}$ One team of autistic people, people with intellectual, physical, or sensory disabilities, and people without disabilities wished to examine associations between violence, disability and health among people with developmental disabilities. ${ }^{34}$ Nicolaidis and colleagues selected commonly used measures of depression, stress, PTSD symptoms, somatic symptoms, and social support to include in a survey. The original measures' text-based Likert scales were flagged by some members of their team as confusing. Therefore, they changed the wording of response options to be more precise and added graphic representations of response items indicating frequency and/or valence. For example, a 5-point Likert scale with response items ranging from "none of the time" to "all of the time" was represented by five cylinders of incrementally increasing fullness. Items evaluating satisfaction were adapted to include graphic 
smiley faces with "very much satisfied" represented by a simple full smile and "not at all satisfied" with a simple frown face. When the adapted measures were used with a large sample of people with developmental disabilities, the internal inconsistencies of all measures but one were good to excellent.

A second research group collaborated with eight youth with developmental disabilities to develop the Pediatric Disability Inventory-Patient Reported Outcome. ${ }^{35}$ Researchers and youth group members examined and defined the construct of "functional performance" to reflect the needs and experiences of people with developmental disabilities, identified tasks to include within the assessment, and refined items. This process appeared to be substantially less participatory than the process developed by Nicolaidis and colleagues ${ }^{34}$ as the youth did not play a role in developing the research question, selecting measures, or dissemination. However, pictures emerged as a useful tool to help the youth engage, aligning with Nicolaidis and colleagues' findings.

Inspired by prior participatory research and associated calls to prioritize autistic voices and intellect in autism research to increase its social validity, ${ }^{36-38}$ our research team has committed to conducting research using participatory methods which include autistic and nonautistic researchers and community members in research conceptualization, design, and dissemination. As discussed above, adapting purely text-based response options to include pictures is a key strategy for including more diverse autistic individuals in dialogue about their experiences, supports, and research. In the current study, we develop a multimodal (comprised of pictures and words) emotion rating measure that begins to capture the multidimensionality of emotions. Our work expands upon research using unidimensional picture-based response options assessing valence, such as the smiley faces used by Nicolaidis and colleagues, ${ }^{34}$ by providing 
opportunities for people with diverse communicative needs to express a greater range of emotions.

\section{Study Aims and Overview}

The overarching aim of the current study was to develop an intuitive and humanistic emotion rating scale, inspired by the original SAM, which can be used for research purposes without (or with) written or verbal instructions for autistic and non-autistic participants. To achieve this overarching aim, we had three sub-aims:

1. Develop a new multidimensional emotion rating measure (the AAPE)

2. Revise the AAPE guided by initial evaluations

3. Use the AAPE in an applied setting

Methods and findings will be presented in four sections: The Participatory Approach section will orient readers to the values underlying this research and the strategies we used to align our work with these values. Next, we provide the methods and results for each aim. Each aim builds on the prior aim; we present methods and results for each aim in the order they were carried out to mirror our iterative research process. This project was approved by the Institutional Review Board at the City University of New York.

\section{Methods}

\section{Participatory Approach}

The research activities described in this paper were conducted by a participatory group of autistic and non-autistic researchers that included the authors of this paper and a larger group that collaborated more distally in this and other research initiatives. Authors of this work include a non-autistic doctoral candidate (AR), two autistic undergraduates (JDS, the artist who drew the AAPE, and AJ, who was involved with conceptualizing the energy dimension and revising the 
artist's renderings), an autistic academic (SKK), and two non-autistic academics (KGL and DD). Group members could join meetings in-person or virtually (depending on their location and/or preference) held once every 6 weeks. People who joined virtually did so via Skype using whichever method of communication they preferred (audiovisual video, voice-only, text-only, or some combination). For all qualitative data described in this paper, coding was conducted by AR and JDS. Coders achieved greater than $80 \%$ agreement on at least $20 \%$ of participant responses in each code category.

The aims of this study were initially developed by AR and KGL (neither of whom is autistic), after viewing the aforementioned poster using the SAM with Deaf clients, as a direction for AR's doctoral dissertation. The overarching idea for this study built on discussions between SKK and KGL about the need for methods to reach autistic people who do not communicate through spoken and/or written language after they developed a study about autistic identity when they were both graduate students and found that it lacked representation by non-speaking people. $^{2}$

The idea to assess and adapt the SAM for use with autistic participants was then shared with the participatory research group. Group members expressed strong support for the idea, indicating interest in developing new visuals to represent each emotion. JDS, an autistic thencollege student who is an artist and worked on this project as part of his successful honors thesis, agreed to draw the scale adaptations. Iterations of each dimension were shared at participatory research meetings where all group members could review and critique elements of each dimension (such as face shape, facial expression, body position, etc.) and suggest changes. In an effort to be transparent about our methods and experiences, Table 1 includes comments from 
autistic participatory group members about their feelings about the research process and our communication as a research group.

\section{[Insert Table 1]}

\section{Methods Aim 1: Developing a New Multidimensional Emotion Rating Measure}

We speculated that more intuitive (e.g., recognizably human) drawings than the SAM would be necessary to increase accessibility. We created two separate dimensions of arousal to measure both positive arousal (neutral to joyful) and negative arousal (neutral to anxious). An adapted scale for dominance, interpreted by this group as social dominance/confidence, was also constructed. Discussions within our research group resulted in multiple iterations of the picturebased adaptations to the SAM over the course of one year. Decisions around which emotions to include and how best to portray them using a humanistic, yet gender- and racially neutral, character emerged through extensive dialogue. Figure 2 depicts the process of iteratively adapting the AAPE. Table 2 depicts key insights of our group members throughout this process.

\section{[Insert Figure 2]}

\section{[Insert Table 2]}

We used a computer-based survey to evaluate interpretability with minimal instructions of an unlabeled version of the SAM (Figure 1) and an unlabeled initial version of the AAPE (Figure 2; initial AAPE dimensions highlighted in gold). Participants were asked, "What do you think these pictures are measuring?" about each of the three SAM scales (valence, arousal, and dominance) and four initial AAPE scales (affect, positive arousal, negative arousal, social confidence) with no further instructions. At this stage, we presented the images without accompanying text to evaluate their interpretability without reliance on text-based scaffolding. Our aim was not to evaluate the validity of the SAM, as we did not use the extensive 
verbal instructions that are supposed to accompany the SAM to clarify its meaning. Instead, our aim was to establish the degree to which the SAM and the initial AAPE were accessible as nonverbal emotion rating measures.

Autistic high school students $\left(n=15, M_{\text {age }}=15.8\right.$ years, $93.3 \%$ male, $46.7 \%$ White/Caucasian, 20\% Hispanic/Latinx, 20\% Black, 20\% Asian/Pacific Islander) were recruited from an informal educational program and autistic college students $\left(n=16, M_{\text {age }}=21.7\right.$ years, 87.5\% male, 37.5\% White/Caucasian, 12.5\% Hispanic/Latinx, 12.5\% Black, 18.8\%

Asian/Pacific Islander, 18.8\% nor reported) were recruited from a mentorship program at a large, public, urban college. Autism classifications were ascertained by parent-report for high school participants and self-report and Individualized Education Plan documentation for college participants in this wave of data collection. Non-autistic students $(n=197)$ were recruited from the Psychology subject pool at the same college.

\section{Results: Aim 1 - Developing a New Multidimensional Emotion Rating Measure}

Among the combined 31 autistic high school and college students who completed the pilot survey, the unlabeled SAM dimension of valence (Figure 1A) was most likely to be understood, with $81 \%$ of students correctly reporting that this scale measures moods, feelings, or level of happiness. Only 19\% of students described a construct relating to "arousal" (Figure 1B) when presented with the scale; many participants reported that the man was hungry or hurt. Only $6 \%$ of students correctly described the SAM scale for "dominance" (Figure 1C) as the authors intended. Instead, students believed the dominance dimension represented body size or physical changes. Non-autistic college students interpreted the SAM with slightly improved accuracy: $93 \%$ demonstrated understanding of valence and $28 \%$ understood arousal. Yet only $16 \%$ understood the dominance dimension. The dominance dimension of the SAM also exhibited 
questionable convergent validity in the original paper establishing the measure, even though textbased supports to guide interpretation were provided in that study. ${ }^{28}$ This data indicated that the SAM is not accessible without accompanying verbal or text-based clarifications.

Data suggested that our adapted multidimensional emotion rating measure (the initial AAPE) was more accessible than the unlabeled SAM. Most of the 31 autistic participants (96.8\%) correctly described the affect scale of the AAPE as depicting emotions, feelings, or moods. The scales measuring positive and negative arousal were similarly well recognized with $90.3 \%$ and $96.8 \%$ of the autistic participants correctly describing the emotions depicted in these scales, respectively. Participants used words such as "stressed" and "anxiety" to describe negative arousal and "joy" or "excitement" to describe the positive arousal dimension. However, only $58.1 \%$ of the participants correctly described the dominance/social confidence scale using terms such as "shyness" and "confidence". This is a significant improvement when compared to the $6 \%$ of autistic participants who were able to correctly describe the "dominance" dimension of the SAM, but still below optimal for use in future research as intended.

\section{Methods: Aim 2 - Data-Informed Iteration of Multidimensional Emotion Rating Measure \\ Given the sub-optimal results for the dominance/social confidence dimension of the} AAPE, we eliminated the dominance dimension at this stage. We decided instead to include dimensions depicting emotions that autistic members of our team felt would be most relevant to our long-term goal of using our emotion rating scale to evaluate autistic identity (to be described in a future publication). Based on previous research concerning camouflaging of autistic behaviors and the large amounts of energy autistic individuals report dedicating to camouflaging, ${ }^{5}$ we decided that dimensions of shame to pride and depleted to high energy would be useful. Camouflaging in this case means to engage in behavior that aims to mask or hide parts 
of oneself from others in an attempt to "pass" when interacting socially. The decision to focus on energy was sparked by AJ's personal experiences with camouflaging contributing to low energy. Sketches of these new dimensions are depicted in Figure 2 with accompanying examples of the decision-making process in Table 2.

We evaluated the final iteration of our Multidimensional Scale Assessing Affect, Anxiety, Pride and Energy, the AAPE, as an aspect of a broader online survey about the experiences and identities of autistic postsecondary students. A total of 72 students completed this survey with an average age of 24.03 years $( \pm 6.9)$. Students were $40.3 \%$ male, $43.1 \%$ female, $13.9 \%$ non-binary, with $2.8 \%$ reported "agender" or "mostly male". Students in this sample were 76.4\% White/Caucasian, 6.9\% Hispanic/Latinx, 9.7\% Asian, and less than 5\% Indigenous ( $n=3)$, Black ( $n=2)$, Pacific Islander $(n=2)$, Middle Eastern/North African $(n=1)$, or preferred not to answer $(n=3)$. Participants were eligible if currently enrolled as a university student (certificate through doctoral level, full or part-time) and if they had an autism diagnosis (verified by selfreport). Participants represented 9 different countries ( $n=47$ from the United States, $n=15$ from the United Kingdom, $n=10$ from other countries).

Participants were presented with the final AAPE emotion scales and asked, "What do you think these pictures are showing?" They were then told what the emotion for each particular scale was intended to portray and asked to "Please rate how much you agree that these pictures show (intended emotion)" on a 5-point scale from strongly disagree (1) to strongly agree (5). Affect was described using the labels "sad to joyful", anxiety using "calm to anxious", pride using "ashamed to proud", and energy using "low energy to high energy".

Results: Aim 2 - Data-Informed Iteration of Multidimensional Emotion Rating Measure 
Coding of open-ended responses to the unlabeled dimensions indicated that our four emotion scales were generally well-understood by autistic college students in our sample. Consistent with previous results, the scale for emotional valence performed best with $97.2 \%$ of the 72 participants interpreting the scale correctly. Our scale of negative arousal was also generally interpretable with $79.2 \%$ of participants correctly interpreting the scale. The new scale for energy level performed well; $84.7 \%$ of participants correctly interpreted it. Similar to the limitations of the original SAM dominance dimension and our revised dominance/social confidence dimension, our new shameful to prideful dimension performed the worst with $52.8 \%$ of participants correctly describing the intended construct. Participants who misinterpreted the scale for pride described the dimension as anxious or worried to calm $(n=10)$ or afraid $(n=4)$, and nine participants responded with "I don't know" when asked to describe the dimension.

When participants were informed what the scales were intended to depict, their quantitative ratings were better than average for all dimensions. Participants generally agreed that affect $(M=4.28, S D=.92)$ and negative arousal $(M=4.29 \pm .86)$ depicted the intended emotional dimension, followed by energy level $(M=4.08 \pm 1.00)$ and pride $(M=3.50 \pm 1.20)$. It appears that simple text-based instructions indicating the poles of each dimension may help participants understand them. Findings suggest that the pride dimension requires further revisions before being used without accompanying explanatory language.

\section{Initial Application of AAPE in Technology Camp for Neurodivergent Youth}

In summer 2019, we used the final AAPE to provide youth in an informal technology education program with opportunities to share how they felt about different instructional strategies. The summer program is delivered through 30-hour, week-long sessions, with separate programs for children (7-12 years) and adolescents (13 to 20 years). 
Fifty students whose parents confirmed they had an autism diagnosis $(25$ children $M_{\mathrm{age}}=9.9, S D=1.6$ and 25 adolescents $\left.M_{\mathrm{age}}=15.9, S D=2.1\right)$ completed brief online surveys at the end of each week. There were asked: "How did you feel when your teacher asked you to [teaching strategy] this week? The teaching strategies assessed included: "play games", “group activities", “activities on your own”, "taught the class by talking to everyone”, and "showed you how to do something and then let you do it yourself" After each prompt, the four unlabeled dimensions of the final AAPE were presented (affect, anxiety, pride and energy). Based on observation and informal conversations, students appeared much more willing to complete the survey with the AAPE than they had been over the prior three summers when we had used purely text-based surveys.

The internal consistency of the AAPE, or degree to which positive affect, low anxiety, energy and pride aligned with one another, were generally acceptable for all activities in the full sample. However, internal consistencies tended to be higher for children than adolescents: games $\alpha=.70$ (child $\alpha=.83$; adolescent $\alpha=.53$ ), group activities $\alpha=.75$ (child $\alpha=.87$; adolescent $\alpha=.48$ ), individual activities $\alpha=.85$ (child $\alpha=.92$; adolescent $\alpha=.71$ ), teacher talking $\alpha=.82$ (child $\alpha=.79$; adolescent $\alpha=.84$ ), and doing it yourself $\alpha=.81$ (child $\alpha=.89$; adolescent $\alpha=.64$ ). The difference in internal consistency between children and adolescents may reflect heightened distractibility among adolescents which, anecdotally, has been observed by our research team and/or the more complicated (and sometimes contradictory) emotions that adolescents experience relative to children. ${ }^{39}$ The adolescent classroom at this organization also serves students across a wider age range when compared to the child-age classroom and this variability may also impact the AAPE's internal consistency. 
A repeated measures analysis with age (adolescent versus child) as a between subjects factor revealed differences in the affective dimension across activities, $F(1,48)=3.15, p=.016$, and no interaction with age $(p=.15)$. Follow up analyses revealed that games elicited more positive affect than group activities and the teacher talking $(p s<.01)$. An analysis with anxiety as the outcome measure revealed differences in anxiety, $F(1,48)=5.15, p=.03$, and no interaction $(p=.54)$. Students rated the teacher talking as more anxiety provoking than games and solitary activities $(p \mathrm{~s}<.04)$. A similar analysis revealed differences in energy, $F(1,48)=$ $2.68, p=.03$, and no interaction $(p=.51)$. Students found games more energizing than the teacher talking and group activities $(p s<.04)$. Doing it yourself was also more energizing than the teacher talking $(p=.05)$. Consistent with the limitations in the unlabeled pride dimension observed when addressing Aim 2, no contextual differences were observed for pride, $p=.16$. Students were also asked if they would like to return to the camp in the future. Heightened positive affect, pride and energy during games and group activities, energy during solo activities, and positive affect in response to the teacher talking were all associated with heightened desire to return $(p \mathrm{~s}<.04)$.

\section{Discussion}

This paper presents the development, iteration, and initial evaluation of a novel, picturebased, multidimensional measure of emotions developed by a participatory group of autistic and non-autistic scholars. Three of the dimensions (i.e., affect, anxiety, and energy level) received acceptable ratings for future research at this time, while the fourth dimension may require further adaptations to more clearly capture the construct of "pride". This dimension depicts the most complex emotion chosen by our group and required the most extensive editing during development. We experienced the most disagreements among group members on how to best 
portray this range of emotions and spent significant time editing character elements such as hand, eye, head, shoulder position, and facial expressions.

While there may be room for improvement in how pride is portrayed within the AAPE, difficulties recognizing complex emotions, specifically those that index social norms, may also be a reason for decreased comprehension of this dimension. Previous research on emotion recognition in autistic people suggests that understanding complex emotions may be more difficult than understanding the more straightforward emotions portrayed by our other dimensions of the AAPE that elicited better comprehension. ${ }^{40}$ Indeed, prior research suggests that autistic children may experience specific difficulty recognizing embarrassment and shame relative to non-autistic children. ${ }^{40}$ The authors attributed this difficulty to reduced sensitivity to violations of social norms. They did not, however, assess for potential alexithymia. As seen in some open-ended responses from participants who cited other emotions such as anxiety or fear when asked to describe the pride dimension and as noted by an autistic reviewer of this manuscript, the experiences of shame and pride may differ between autistic and non-autistic people. This dimension may also be difficult to identify without a specific situation to react to.

A follow-up study comparing interpretability of the AAPE among autistic and nonautistic students may help to disambiguate the reasons why the pride dimension proved hardest to capture. We recommend that researchers include the pride dimension in their future research but that they also ask participants to share what each unlabeled dimension means to them and then provide text-based labels to ensure the dimension is interpreted as intended (see Appendix A). Providing open-ended opportunities for participants to interpret and critique measures is a valuable extension upon participatory research partnerships as it allows participants to also contribute to measure refinement and evaluation of the validity of research methods. 
Overall, the AAPE makes a significant contribution to the current emotion literature and has potential for applications beyond autism research. Our initial application of the AAPE with autistic children and adolescents in an educational program suggests that it is engaging, generally has acceptable internal consistency (particularly for children), and is informative. As expected, autistic youth's ratings of instructional practices using the AAPE largely aligned with the central premise of Universal Design, that learners prefer multimodal activities rather than just being talked to. ${ }^{41}$

We also believe that documentation of our participatory research process throughout this study, including a discussion of all authors' contributions from idea generation to data analysis and reporting, and the inclusion of autistic authors' voices when reflecting on the overall research processes, represents a move towards a fully transparent participatory model that we hope will be replicated in future publications. We are also learning as a group how to better accommodate diverse communication styles and executive functioning challenges (which are not specific to autistic collaborators). Difficulties with technology during virtual meetings, more advance planning, and clearer documentation of the decision-making process are areas our group has been working to improve. While it is promising that research groups are increasingly moving towards participatory models guided by helpful recommendations from more established groups, ${ }^{38}$ papers often do not yet include this level of detail when describing participatory models. $^{42}$

\section{Limitations}

While results are promising and indicate the AAPE has utility, there are limitations in sampling and methodology. We conducted initial assessments of construct validity but did not systematically assess convergent and divergent validity. The use of both contextually situated 
and broader online samples is a strength of this study. However, the racial and ethnic diversity of participants was limited. The AAPE should be assessed with a more diverse sample and in relation to other measures in order to confirm its validity. Autism diagnoses for participants were based on either parent-report (for children and adolescents) or self-report (for the majority of college students who participated in this study); our inability to independently verify diagnoses is a limitation of this study.

An original goal of this study was to develop a scale that could be used without any written or verbal prompts to assess emotions and autistic identity with non-speaking autistic people. After evaluating the degree to which the dimensions were interpretable without text, we recommend incorporating text-based prompts, so as not to alienate people by assuming a false equivalence between non-speaking and low literacy. Unfortunately, we have not yet been successful in our attempts to recruit non-speaking participants, so do not yet know if the AAPE will be interpretable or reliable for non-speaking people. It is possible that the AAPE may require additional iterations or supports to be suitable for a population with more diverse communicative strengths and challenges. We hope to expand our participatory process to include non-speaking individuals in future iterations to ensure that our measure will function as intended. We believe it is essential to take these steps in our future research to ameliorate the gap in the literature surrounding autistic identity in non-speaking people.

\section{Conclusions and Future Directions}

The AAPE was well-received by participants and has utility for future research studies. Additional editing may be required to represent more complex emotions, namely pride, before using this particular dimension in future research, especially without accompanying text. 
Additional testing is needed to assess feasibility, validity, and reliability of the AAPE with people with more diverse communication skills.

In reflecting upon our overarching aim, to develop a multimodal emotion rating scale to provide autistic people with diverse needs and skills with a tool they can use to express emotions, we have thus far developed a scale with a participatory group of autistic and non-autistic researchers and tested our scale with both autistic college students and school-aged participants. Internal consistencies were promising for children but show room for improvement when used with adolescents.

As discussed above, we intend to report on the use of the AAPE to rate picture-based depictions of autistic identity in a future publication. Work with autistic people has characterized the experience of being autistic and the ways autistic people make sense of the autism label. ${ }^{4,43,44}$ Some studies have reported negative experiences associated with autism including feeling the need to camouflage or mask traits to be perceived as normal by society, social groups, and employers 5,45 while others describe strengths associated with autism such as strong memories, advanced knowledge, and systematic thinking. ${ }^{46}$ These studies have provided a glimpse into the lived experiences of autistic people. However, specific emotions associated with these experiences and the degree to which these experiences are shared with autistic people who do not communicate through the language-based methodologies used in these studies remains unclear. The AAPE is a promising tool to begin to address this lacuna in the literature. The AAPE, available open-access from any author of this report (see appendix A), is accessible and engaging and has utility for people with diverse communicative preferences. 


\section{Acknowledgements}

We would like to acknowledge Emmanuel Garcia-Lesy whose work adapting the SAM with

Deaf individuals sparked the idea for the current study and who provided initial feedback on the study design. We would also like to acknowledge Beth Rosenberg for her assistance collecting data with children and teens using the AAPE and the many students who provided feedback on study measures throughout this process. 


\section{References}

1. Fletcher-Watson S, Happé F. Autism: A New Introduction to Psychological Theory and Current Debate. Routledge; 2019.

2. Kapp SK, Gillespie-Lynch K, Sherman LE, Hutman T. Deficit, difference, or both? Autism and neurodiversity. Dev Psychol. 2013;49(1):59-71. doi:10.1037/a0028353

3. Kenny L, Hattersley C, Molins B, Buckley C, Povey C, Pellicano E. Which terms should be used to describe autism? Perspectives from the UK autism community. Autism. 2016;20(4):442-462.

4. Jones RSP, Huws JC, Beck G. 'I'm not the only person out there': insider and outsider understandings of autism. Int J Dev Disabil. 2013;59(2):134-144. doi:10.1179/2047387712Y.0000000007

5. Hull L, Petrides KV, Allison C, et al. "Putting on My Best Normal": Social Camouflaging in Adults with Autism Spectrum Conditions. J Autism Dev Disord. 2017;47(8):2519-2534. doi:10.1007/s10803-017-3166-5

6. Mason D, McConachie H, Garland D, Petrou A, Rodgers J, Parr JR. Predictors of quality of life for autistic adults: Quality of life of autistic adults. Autism Res. 2018;11(8):1138-1147. doi:10.1002/aur.1965

7. Gotham K, Marvin AR, Taylor JL, et al. Characterizing the daily life, needs, and priorities of adults with autism spectrum disorder from Interactive Autism Network data. Autism. 2015;19(7):794-804. doi:10.1177/1362361315583818

8. Jack A, A. Pelphrey K. Annual Research Review: Understudied populations within the autism spectrum-current trends and future directions in neuroimaging research. $J$ Child Psychol Psychiatry. 2017;58(4):411-435.

9. Stedman A, Taylor B, Erard M, Peura C, Siegel M. Are children severely affected by autism spectrum disorder underrepresented in treatment studies? An analysis of the literature. J Autism Dev Disord. 2019;49(4):1378-1390.

10. Nicholas DB, Orjasaeter JD, Zwaigenbaum L. Considering Methodological Accommodation to the Diversity of ASD: A Realist Synthesis Review of Data Collection Methods for Examining First-Person Experiences. Rev J Autism Dev Disord. 2019;6(2):216-232.

11. Tesfaye R, Courchesne V, Yusuf A, et al. Assuming ability of youth with autism: Synthesis of methods capturing the first-person perspectives of children and youth with disabilities. Autism. 2019;23(8):1882-1896.

12. Kaldy Z, Giserman I, Carter AS, Blaser E. The mechanisms underlying the ASD advantage in visual search. J Autism Dev Disord. 2016;46(5):1513-1527. 
13. Dubi K, Schneider S. The Picture Anxiety Test (PAT): A new pictorial assessment of anxiety symptoms in young children. J Anxiety Disord. 2009;23(8):1148-1157.

14. Hartley SL, MacLean Jr WE. A review of the reliability and validity of Likert-type scales for people with intellectual disability. J Intellect Disabil Res. 2006;50(11):813-827.

15. Rand S, Towers A-M, Razik K, et al. Feasibility, factor structure and construct validity of the easy-read Adult Social Care Outcomes Toolkit (ASCOT-ER). J Intellect Dev Disabil. 2019:1-14.

16. Turnpenny A, Caiels J, Whelton B, et al. Developing an easy read version of the adult social care outcomes toolkit (ASCOT). J Appl Res Intellect Disabil. 2018;31(1):e36-e48.

17. Fogel A. A relational perspective on the development of self and emotion. Identity Emot Dev Self-Organ. 2001:93-114.

18. Greenspan SI, Shanker S. The First Idea: How Symbols, Language, and Intelligence Evolved from Our Primate Ancestors to Modern Humans. Da Capo Press; 2009.

19. Eack SM, Mazefsky CA, Minshew NJ. Misinterpretation of facial expressions of emotion in verbal adults with autism spectrum disorder. Autism. 2015;19(3):308-315. doi:10.1177/1362361314520755

20. Golan O, Gordon I, Fichman K, Keinan G. Specific patterns of emotion recognition from faces in children with ASD: Results of a cross-modal matching paradigm. J Autism Dev Disord. 2018;48(3):844-852.

21. Macdonald H, Rutter M, Howlin P, et al. Recognition and expression of emotional cues by autistic and normal adults. J Child Psychol Psychiatry. 1989;30(6):865-877.

22. Kanner L. Autistic disturbances of affective contact. Nerv Child. 1943;2(3):217-250.

23. Baron-Cohen S. Do people with autism understand what causes emotion? Child Dev. 1991;62(2):385-395.

24. Gernsbacher MA, Yergeau M. Empirical failures of the claim that autistic people lack a theory of mind. Arch Sci Psychol. 2019;7(1):102.

25. Brewer R, Murphy J. People with autism can read emotions, feel empathy. Sci Am. $2016 ; 12$.

26. Poquérusse J, Pastore L, Dellantonio S, Esposito G. Alexithymia and autism spectrum disorder: a complex relationship. Front Psychol. 2018;9.

27. \#Alexithymia isn't only a problem of not understanding our emotions - I CAN Be Autistic. https://visualvox.wordpress.com/2017/03/26/alexithymia-isn't-only-a-problem-of-notunderstanding-our-emotions/. Accessed December 30, 2019. 
28. Bradley MM, Lang PJ. Measuring emotion: The self-assessment manikin and the semantic differential. J Behav Ther Exp Psychiatry. 1994;25(1):49-59. doi:10.1016/00057916(94)90063-9

29. Bynion T-M, Feldner MT. Self-assessment manikin. Encycl Personal Individ Differ. 2017:1-3.

30. Young CB, Raz G, Everaerd D, et al. Dynamic Shifts in Large-Scale Brain Network Balance As a Function of Arousal. J Neurosci. 2017;37(2):281-290. doi:10.1523/JNEUROSCI.1759-16.2016

31. Bölte S, Feineis-Matthews S, Poustka F. Brief Report: Emotional Processing in HighFunctioning Autism-Physiological Reactivity and Affective Report. J Autism Dev Disord. 2008;38(4):776-781. doi:10.1007/s10803-007-0443-8

32. Garcia-Lesy, Emmanuel. "Deaf Friendly" Emotion Assessment with the Self-Assessment Manikin: Case Report. In: ; 2017.

33. Lang PJ, Bradley MM, Cuthbert BN. International affective picture system (IAPS): Technical manual and affective ratings. NIMH Cent Study Emot Atten. 1997;1:39-58.

34. Nicolaidis C, Raymaker D, Katz M, et al. Community-Based Participatory Research to Adapt Health Measures for Use by People With Developmental Disabilities. 2015;9:14.

35. Kramer JM, Schwartz AE. Development of the Pediatric Disability Inventory-Patient Reported Outcome (PEDI-PRO) measurement conceptual framework and item candidates. Scand J Occup Ther. 2018;25(5):335-346. doi:10.1080/11038128.2018.1502344

36. Fletcher-Watson S, Adams J, Brook K, et al. Making the future together: Shaping autism research through meaningful participation. Autism. 2018:1362361318786721.

37. Pellicano E, Dinsmore A, Charman T. What should autism research focus upon? Community views and priorities from the United Kingdom. Autism. 2014;18(7):756-770.

38. Nicolaidis C, Raymaker D, Kapp SK, et al. The AASPIRE practice-based guidelines for the inclusion of autistic adults in research as co-researchers and study participants. Autism. 2019:1362361319830523.

39. Fischer KW, Shaver PR, Carnochan P. How emotions develop and how they organise development. Cogn Emot. 1990;4(2):81-127.

40. Capps L, Yirmiya N, Sigman M. Understanding of simple and complex emotions in nonretarded children with autism. J Child Psychol Psychiatry. 1992;33(7):1169-1182.

41. Burgstahler S. Universal design: Implications for computing education. ACM Trans Comput Educ TOCE. 2011;11(3):19. 
42. Jivraj J, Sacrey L-A, Newton A, Nicholas D, Zwaigenbaum L. Assessing the influence of researcher-partner involvement on the process and outcomes of participatory research in autism spectrum disorder and neurodevelopmental disorders: A scoping review. Autism. 2014;18(7):782-793.

43. Humphrey N, Lewis S. 'Make me normal': The views and experiences of pupils on the autistic spectrum in mainstream secondary schools. Autism. 2008;12(1):23-46. doi:10.1177/1362361307085267

44. Mogensen L, Mason J. The meaning of a label for teenagers negotiating identity: experiences with autism spectrum disorder. Sociol Health Illn. 2015;37(2):255-269. doi:10.1111/1467-9566.12208

45. Cage E, Troxell-Whitman Z. Understanding the Reasons, Contexts and Costs of Camouflaging for Autistic Adults. J Autism Dev Disord. January 2019. doi:10.1007/s10803-018-03878-X

46. Russell G, Kapp SK, Elliott D, Elphick C, Gwernan-Jones R, Owens C. Mapping the Autistic Advantage from the Accounts of Adults Diagnosed with Autism: A Qualitative Study. Autism Adulthood. 2019;1(2):124-133. 


\section{Appendix A}

Images used in the Multidimensional Visual Scale Assessing Affect, Anxiety, Energy, and Pride (AAPE)

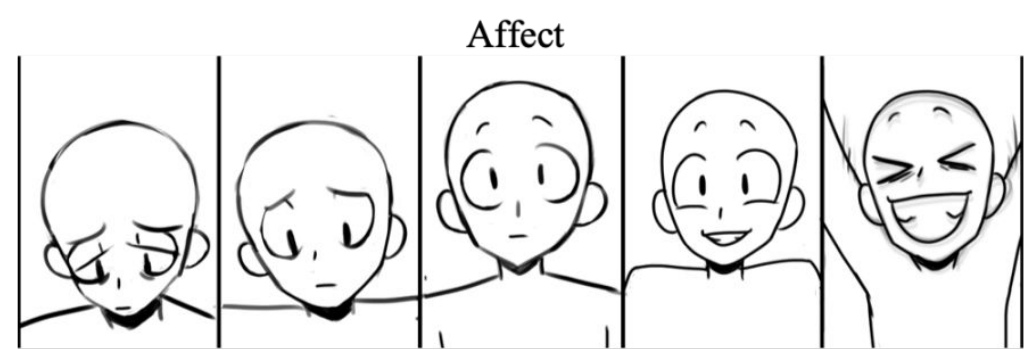

Sad

Joyful
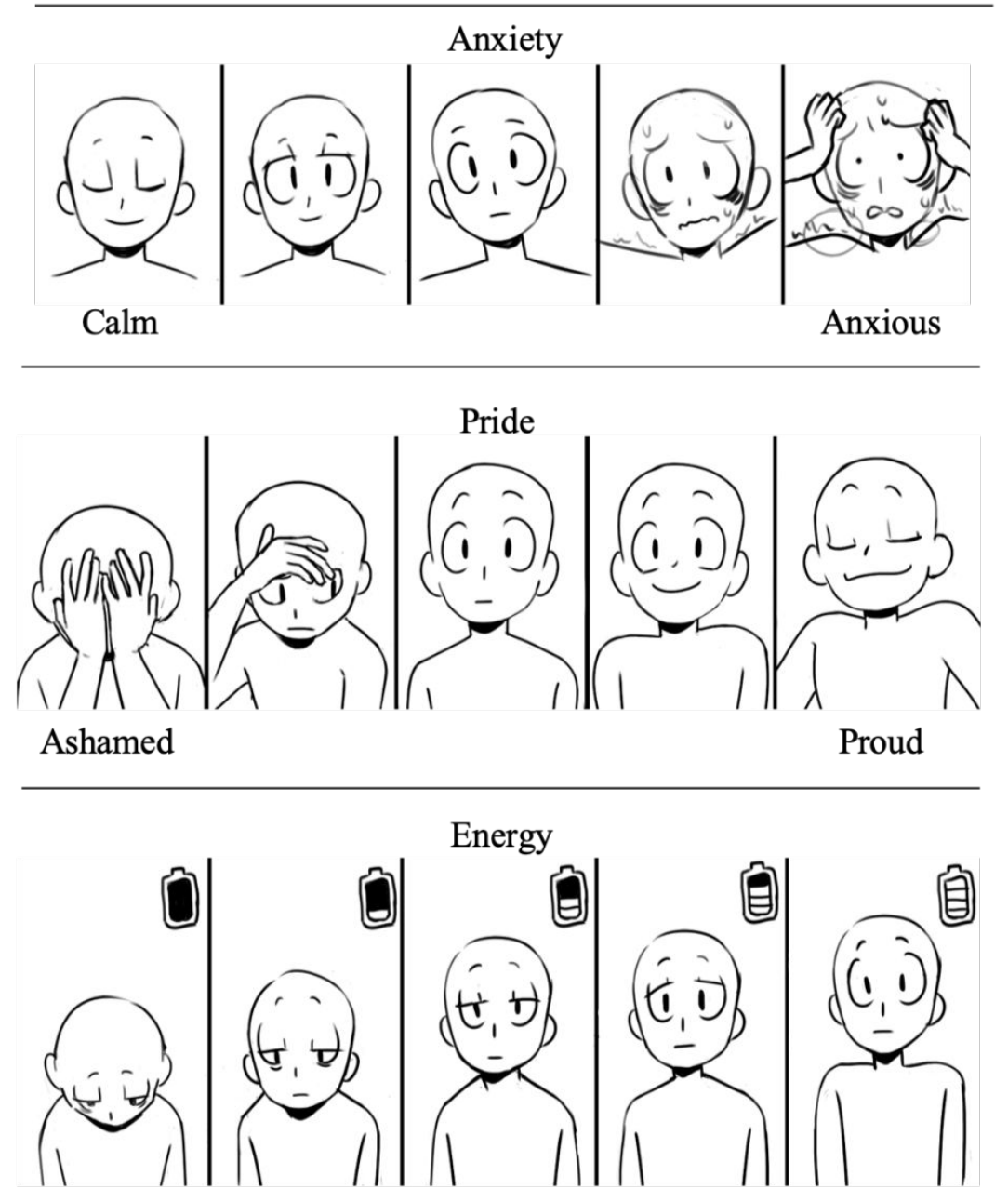

Low energy

High energy

Scoring guidelines:

Our research team currently scores the AAPE numerically by assigning a value from 1-5 for each emotion response option from left to right, with the exception of Anxiety which is reverse scored. For example, joyful, calm, proud, and high energy each receive a score of 5 while sad, anxious, ashamed, and low energy each receive a score of 1. 
"Why was this study done?"

There are very few tools that autistic people can use to share how they feel. We wanted to develop a tool to help autistic people express their emotions using pictures. Pictures can help autistic people share how they feel.

"What was the purpose of this study?"

We wanted to make an easy-to-understand tool that autistic people can use to share how they feel.

"What did the researchers do?"

Our research group is participatory, meaning that autistic and non-autistic researchers worked together to make our tool. An autistic artist drew the tool. We called it the AAPE, which stands for the emotions it assesses: Affect, Anxiety, Pride and Energy. We worked together to see how well the AAPE worked. We used a survey to see if autistic high school and college students understood our first try at the AAPE and we learned how to make the AAPE better from these students. We worked together to make the AAPE better. Then we did another survey with autistic college students to see if our second try at the AAPE worked better. Then we asked autistic kids and teenagers to use the AAPE to share how they felt about different ways of teaching.

"What were the results of the study?"

In our final test, we asked 72 autistic college students to tell us what emotions they thought the AAPE was showing. College students thought that affect (97.2\%), anxiety (79.2\%), and energy $(84.7 \%)$ showed the emotions we aimed to show with room for improvement in how pride (52.8\%) was shown. After we told participants which emotion each scale was showing, they agreed that affect (average score 4.28 out of 5) and anxiety (4.29 out of 5) showed the emotions best, followed by energy ( 4.08 out of 5 ) and pride ( 3.5 out of 5 ) on a scale from one to five (strongly agree).

Results from our study with children and teens found that students preferred using the AAPE compared to text-based surveys we used in the past. These results also showed that the AAPE does a good job at measuring emotions in this setting. Edits may still be needed to better show "pride". Work is needed with non-speaking people to make sure our measure works well for people who communicate in different ways.

"What are potential weaknesses in the study?"

Right now, we have only tested the AAPE with autistic college students using an online survey. This sample of college students was not very diverse, and we did not include participants who communicate using different methods. We plan to use the AAPE with more diverse groups in future studies.

"How will these findings help autistic adults now or in the future?"

In a future study, we will use the AAPE to rate pictures that show experiences of being autistic like feeling outside of a social group, having sensory difficulties, and making patterns. We would like to do this because some studies talk about negative experiences of autism like feeling the 
need to hide autistic traits, but other studies describe strengths of autism like strong memories and advanced knowledge in particular subjects. However, these studies do not talk about the emotions that come with these experiences and if these experiences are shared with autistic people who do not use speech to communicate. The AAPE is a tool that might help us address this research question. 
Figure 1. The Self-Assessment Manikin, an existing picture-based scale to measure emotional responses to stimuli in three areas: valence (A), arousal (B), and dominance/control (C) ${ }^{13}$

Figure 2. Diagram showing the iterative process of scale development, as inspired by the Self-Assessment Manikin, resulting in a 4-item visual rating scale of emotions. Affect (from negative to positive), negative arousal/anxiety (from calm to anxious), pride (from shameful to prideful), and energy (from depleted to energetic) comprise the completed scales presented in this study. A full description of the items and comments from participatory research group members on the design and iteration process are included in Table 1. 
Figure 1. The Self-Assessment Manikin, an existing picture-based scale to measure emotional responses to stimuli in three areas: valence (A), arousal (B), and dominance/control (C).

$150 \times 102 \mathrm{~mm}(150 \times 150 \mathrm{DPI})$ 
Figure 2. Diagram showing the iterative process of scale development, as inspired by the Self-Assessment Manikin, resulting in a 4-item visual rating scale of emotions. Affect (from negative to positive), negative arousal/anxiety (from calm to anxious), pride (from shameful to prideful), and energy (from depleted to energetic) comprise the completed scales presented in this study. A full description of the items and comments from participatory research group members on the design and iteration process are included in Table 1.

$215 \times 279 m m(150 \times 150$ DPI $)$ 